\title{
The Mystery of CEMPs $+r$ Stars and the Dual Core-Flash Neutron Superburst
}

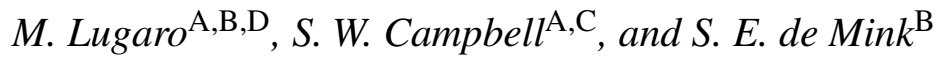 \\ A Centre for Stellar and Planetary Astrophysics, School of Mathematical Sciences, \\ Monash University, Clayton, VIC 3800 \\ B Sterrekundig Instituut, University of Utrecht, Postbus 80 000, 3508 TA Utrecht, The Netherlands \\ C Departament de Física i Enginyeria Nuclear, EUETIB, Universita Politècnica de Catalunya, \\ E-08036, Barcelona, Spain \\ D Corresponding author. Email: Maria.Lugaro@sci.monash.edu.au
}

Received 2008 December 15, accepted 2009 January 15

\begin{abstract}
Carbon-enhanced metal-poor $(\mathrm{CEMP} s+r)$ stars show large enhancements of elements produced both by the slow and the rapid neutron capture processes (the $s$ and $r$ process, respectively) and represent a relatively large fraction, $30 \%$ to $50 \%$, of the CEMP population. Many scenarios have been proposed to explain this peculiar chemical composition and most of them involve a binary companion producing the $s$ process elements during its Asymptotic Giant Branch (AGB) phase. The problem is that none of the proposed explanations appears to be able to account for all observational constraints, hence, alternatives are needed to be put forward and investigated. In this spirit, we propose a new scenario for the formation of CEMP $s+r$ stars based on S. W. Campbell's finding that during the 'dual core flash' in low-mass stars of extremely low metallicity, when protons are ingested in the He-flash convective zone, a 'neutron superburst' is produced. Further calculations are needed to verify if this neutron superburst could make the $r$-process component observed in CEMP $s+r$, as well as their Fe abundances. The $s$-process component would then be produced during the following AGB phase.
\end{abstract}

Keywords: nuclear reactions, nucleosynthesis, abundances — stars: AGB and post-AGB — binaries: general — stars: chemically peculiar — stars: Population II

\section{The Group CEMPs $+r$ Stars}

CEMP $s+r$ stars are Population II stars, i.e. old halo stars with very low abundance of metals $([\mathrm{Fe} / \mathrm{H}]<-2)^{1}$, showing large carbon overabundances of $[\mathrm{C} / \mathrm{Fe}]>1.7$, together with $s$ - and $r$-process enhancements: $[\mathrm{Ba} / \mathrm{Fe}]>1$ and $[\mathrm{Eu} / \mathrm{Fe}]>1$, with $[\mathrm{Ba} / \mathrm{Eu}]>0$. Seventeen CEMP $+r$ stars have been discovered to date, comprising $1 \%$ of all Population II stars and $30 \%$ to $50 \%$ of the CEMP population (Jonsell et al. 2006). Most of them are located at the turn-off point, just leaving the main sequence.

Figure 1 shows the $\mathrm{Ba}$ and $\mathrm{Eu}$ abundances relative to $\mathrm{H}$ observed in stars of Population II, selected to have $[\mathrm{Fe} / \mathrm{H}]<-2$, from the web-based database of Suda et al. (2008). This figure is similar to Figure 7 of Jonsell et al. (2006) except that, while in the figure by Jonsell et al. (2006) the $\mathrm{Ba}$ and Eu abundances are plotted with respect to $\mathrm{Fe}$, we preferred to use $\mathrm{H}$ as the reference because of the large uncertainties affecting the Fe abundance determination in CEMP stars, usually up to 0.5 dex.

Also, our classification into different classes of neutroncapture-enriched stars (see legenda in Figure 1) is a somewhat simplified version of the classification described in

${ }^{1}[\mathrm{X} / \mathrm{Y}]=\log (\mathrm{X} / \mathrm{Y})_{\mathrm{star}}-\log (\mathrm{X} / \mathrm{Y})_{\odot}$.
Table 9 of Jonsell et al. (2006). Fourteen out of the 114 stars shown Figure 1 from the Suda et al. (2008) database are enriched in both $r$ - and $s$-process elements and have $[\mathrm{Ba} / \mathrm{Eu}]>0$. We emphasize that for most systems different abundances are quoted by different authors and thus fractions quoted for CEMP $s+r$ stars should be interpreted with care as they depend on the adopted selection criteria and abundances.

In spite of this difference, both our Figure 1 and Jonsell et al. (2006)'s Figure 7 clearly show that in terms of $\mathrm{Ba}$ and Eu abundances CEMP $+r$ stars are a very homogeneous group, clearly separated from the other classes of Population II stars. First, CEMP $+r$ stars are typically those showing the largest enrichments both in $\mathrm{Ba}$ and in $\mathrm{Eu}$, and as such they do not appear to be a subclass of any of the other groups of neutron-capture stars. Also in terms of the $\mathrm{Fe}$ abundance CEMP $+r$ are quite homogeneous, with $[\mathrm{Fe} / \mathrm{H}]$ centered at -2.55 , and a scatter $\simeq 0.26 \mathrm{dex}$ (Jonsell et al. 2006), an iron abundance higher than that of $r$-rich stars $([\mathrm{Fe} / \mathrm{H}]$ centered at -2.84$)$ and lower than that of $s$-rich stars $([\mathrm{Fe} / \mathrm{H}]$ centered at -2.21$)$.

Second, all the different groups of stars in the plot show a clear correlation between their $\mathrm{Ba}$ and their $\mathrm{Eu}$ enhancements. The $r$-rich stars are believed to have formed from material polluted by a Type II supernova 


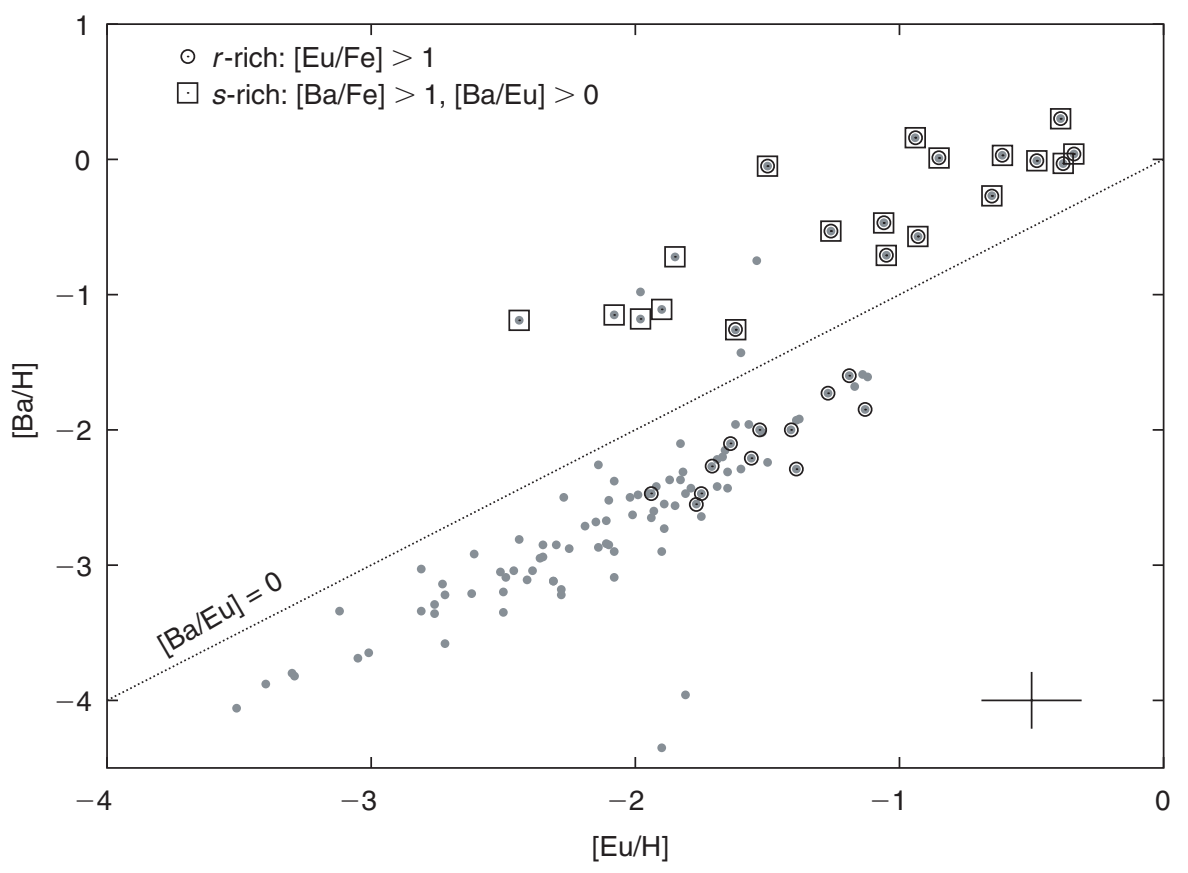

Figure $1 \mathrm{Ba}$ and Eu abundances relative to $\mathrm{H}$ observed in metal-poor stars $([\mathrm{Fe} / \mathrm{H}]<-2)$ as compiled from the web-based database of Suda et al. (2008). The average errorbar is indicated in the lower right corner of the diagram. Systems for which only upper limits are available have been excluded. Different populations of stars showing different enhancements of neutron-capture elements are coded in different symbols, as explained in the legend.

(SNII, e.g. Sneden et al. 1994) and correlate on the same line as the normal Population II stars. This is expected because the $\mathrm{Ba}$ and $\mathrm{Eu}$ abundances in the normal stars are explained as the result of the chemical evolution of the Galaxy, which, in the early times, is entirely dominated by the $r$ process (e.g. Travaglio et al. 1999). Hence, the $\mathrm{Ba}-\mathrm{Eu}$ correlation of normal and $r$-rich stars is made by the $r$ process, which produces most of cosmic Eu $(\simeq 95 \%$ in the solar system), together with a little amount of cosmic Ba ( $\simeq 20 \%$ in the solar system). The $s$-rich stars are believed to have formed by mass transfer from a companion, which evolved through the AGB phase (e.g. Lucatello et al. 2005; Thompson et al. 2008). Also in this case a Ba-Eu correlation is expected by the operation of the $s$ process, which, being the mirror of the $r$ process, produces most of cosmic $\mathrm{Ba}(\simeq 80 \%$ in the solar system) together with the rest of $\mathrm{Eu}(\simeq 5 \%$ in the solar system). Hence, it seems quite straightforward, at least qualitatively, to explain the observed Ba-Eu correlation of the normal Population II stars as well as the $r$-rich and $s$-rich stars. On the other hand, it is not easy to interpret the $\mathrm{Ba}-$ Eu correlation of $s+r$-rich stars as the $s$ process and the $r$ process are not necesserely dependent on each other: the $s$ process is believed to operate in stars of masses below $\sim 10 \mathrm{M}_{\odot}$ during their AGB phase (e.g. Gallino et al. 1998), while the $r$ process is believed to occur in stars of masses above $\sim 10 \mathrm{M}_{\odot}$ during their final SNII explosion. The observed Ba-Eu correlation in $s+r$-rich stars, however, seems to imply that the $s$ - and the $r$-process enrichments should have been somehow connected. The value of the slope of the Ba-Eu correlation of $s+r$-rich stars should be calculated in detail in the future and will provide an important clue to the process that produced the observed correlation.

\section{Scenarios for the Origin of CEMPs $+r$ Stars}

Jonsell et al. (2006) discuss 9 different scenarios to explain the origin of CEMP $s+r$ stars, but none of them is convincing. Here we review and discuss the most popular ones. Favourite scenarios can be classified into two main classes, both invoking mass transfer from a binary companion during its AGB phase. We note in passing that radial velocity monitoring of CEMPs and CEMP $s+r$ are consistent with them being all members of binaries (Lucatello et al. 2005) and that an AGB star companion can also explain the carbon enhancements of CEMP stars, since AGB stars produce large amounts of carbon via partial He burning. AGB star nucleosynthesis is also invoked as the origin of the fluorine overabundances in CEMP stars (Schuler et al. 2007; Lugaro et al. 2008).

In the first class of scenarios the binary system was born out of material contaminated by a nearby SNII, providing the $r$-process elements, and then the primary star evolved into the AGB phase, providing the $s$-process elements via mass transfer to the secondary now observed (see, e.g. Ivans et al. 2005). This scenario can not account for the very large fraction of CEMP $s+r$ among CEMP $s$ stars and the fact that CEMP $+r$ do not appear to be a subgroup, in terms of numbers, of $r$-rich stars, unless formation of binary systems is favoured when star formation is triggered by a supernova, as found by Vanhala \& Cameron (1998). In this context, we note that the idea itself that supernovae may trigger star formation is currently under discussion (see, e.g. Megeath et al. 2008; Mac Low 2008). 
Since in this case the $r$ - and the $s$-process components are independent from each other, it is not clear how this scenario could explain the observed $\mathrm{Ba}-\mathrm{Eu}$ correlation discussed above.

In the second class of scenarios, the primary star in the binary system evolved into the AGB phase, providing the $s$-process elements, and then exploded as a supernova of some kind, providing the $r$-process elements (the 'AGB supernova' scenario). Making an AGB star explode, however, is extremely difficult: only AGB stars of relatively high masses, $>5 \mathrm{M}_{\odot}$, could explode either as electroncapture supernovae, after going through a 'super-AGB' phase where $\mathrm{C}$ burning occurs in the core leaving an $\mathrm{ONe}$ degenerate core (this scenario is discussed in detail by Wanajo et al. 2006), or as supernovae of Type 1.5, when the core grows to reach the Chandrasekhar mass (this scenario is discussed in detail by Zijlstra 2004). However, companion masses in this range would produce more $\mathrm{N}$ than $\mathrm{C}$ during their AGB phase via proton captures at the base of the convective envelope (hot bottom burning, see, e.g. Karakas \& Lattanzio 2007). Hence, these scenarios would produce nitrogen-enhanced metal-poor (NEMP) stars, rather than CEMP stars, unless the $\mathrm{C}$ and $\mathrm{N}$ abundances could be readjusted during the supernova explosion.

In another scenario of this class, the white dwarf left over by the AGB star would produce a supernova via collapse induced by the accretion of material from the secondary star (Qian \& Wasserburg 2003; Cohen et al. 2003). Three separate phases of mass transfer are needed for this scenario to work: (1) transfer of $s$-process AGB material from the primary to the secondary; (2) transfer of mass from the secondary onto the primary evolved into a white dwarf; and (3) transfer of $r$-process enriched material from the primary exploding as supernova onto the secondary. The main problems that make this scenario implausible are that three separated mass transfer phases are quite unlikely and that, since the observed secondary star is, in most cases, just turning off the main sequence, the second phase of mass transfer is problematic as it is difficult to accrete from a distant main sequence star.

Finally, for all of these 'AGB supernova' scenarios it is not actually known if the supernova would be able to produce $r$-process elements as there are no detailed nucleosynthetic models available for any of these cases.

Other scenarios discussed by Jonsell et al. (2006), see also Cohen et al. (2003), include:

- Radiative levitation, dismissed because CEMP $s+r$ stars do not show the same patterns as Ap stars, they are probably not as hot, and have deeper convective zones than Ap stars. Moreover, some CEMP $+r$ are giants with deep convective envelopes where the signature of radiative levitation would be completely erased;

- Triple systems with a SNII and an AGB star, dismissed as these are very unlikely; and

- Hypothetical high neutron-density $s$ process in AGB stars. The few models available on this kind of process give controversial results, and typically seem to exclude this possibility (see Jonsell et al. 2006, for more details and discussion). One problem with this scenario is that the $s$-process distribution, as well as the light element abundances, especially those of $\mathrm{Na}$ and $\mathrm{Mg}$, in the $74 \mathrm{CEMP} s$ and CEMP $+r$ stars observed to date are well fitted by low-mass AGB models $\left(1.2 \mathrm{M}_{\odot}\right.$ to $\left.2 \mathrm{M}_{\odot}\right)$ where the neutron density during the $s$ process is low, being dominated by the ${ }^{13} \mathrm{C}$ neutron source, which operates on a long timescale of $\sim 10^{4} \mathrm{yr}$ (see, e.g. Thompson et al. 2008; Bisterzo 2007). Another constraint that would appear to rule out the hypothesis of a high neutron-density $s$ process in AGB stars comes from spectroscopic observation of ${ }^{151} \mathrm{Eu} /\left({ }^{151} \mathrm{Eu}+{ }^{153} \mathrm{Eu}\right) \simeq 0.6$ in two CEMP $+r$ stars. This ratio is an indicator of the neutron density as it depends on the activation of the branching point at ${ }^{151} \mathrm{Sm}$. The observed ratio indicates neutron densities of the order of $10^{8} \mathrm{~cm}^{-3}$ (Aoki et al. 2003b).

More generally, since the ${ }^{151} \mathrm{Eu} /\left({ }^{151} \mathrm{Eu}+{ }^{153} \mathrm{Eu}\right)$ ratio observed in $r$-rich stars (Sneden et al. 2002; Aoki et al. 2003a) is $\simeq 0.48$, as in the solar system, the value of 0.6 , if confirmed, may be taken as a hint that low neutron-density $s$ process in low-mass AGB stars should have followed the production of the bulk of the Eu, and acted on it by readjusting its isotopic ratios. This may rule out any scenario where the $r$ process follows the $s$ process, i.e. the 'AGB supernova' scenarios.

\section{The Dual Core-Flash Neutron Superburst}

In view of all these difficulties, we propose another scenario for the formation of CEMP $s+r$ stars, based on the finding by Campbell (2007) that a 'neutron superburst' is produced in low-mass stars of extremely low metallicity $\left(<10^{-5}\right)$ during the 'dual core flash' (DCF), i.e. when protons are ingested in the He flash region producing a $\mathrm{H}$ flash. This ingestion has been first reported by Fujimoto, Iben \& Hollowell (1990) and further discussed in Hollowell, Iben \& Fujimoto (1990), Fujimoto, Ikeda \& Iben (2000), Picardi et al. (2004), and Campbell \& Lattanzio (2008). The models of Campbell \& Lattanzio (2008) discussed here show the occurrence of the DCF for masses of 0.85 and $1 \mathrm{M}_{\odot}$ and initial metallicity $Z$ between 0 and $10^{-5}$, i.e. for theoretical low-mass very metaldeficient or metal-free stars, also referred to as Population III, the first stars in the Universe.

Campbell \& Lattanzio (2008) studied in detail the nucleosynthesis during the DCF using a network of 74 species, including neutrons. Figure 2 shows the abundance profiles in the convective $\mathrm{He} / \mathrm{H}$-burning region during the first episode of proton ingestion of the DCF of the stellar model of $0.85 \mathrm{M}_{\odot}$ and initial zero metallicity computed by Campbell (2007). During the flash He burning makes ${ }^{12} \mathrm{C}$, which captures protons producing ${ }^{13} \mathrm{C}$. This nucleus acts as a neutron source via ${ }^{13} \mathrm{C}(\alpha, n){ }^{16} \mathrm{O}$ reactions, with the result that a large number of free neutrons, as well as of ${ }^{16} \mathrm{O}$ nuclei, are produced. The main neutron poison is 

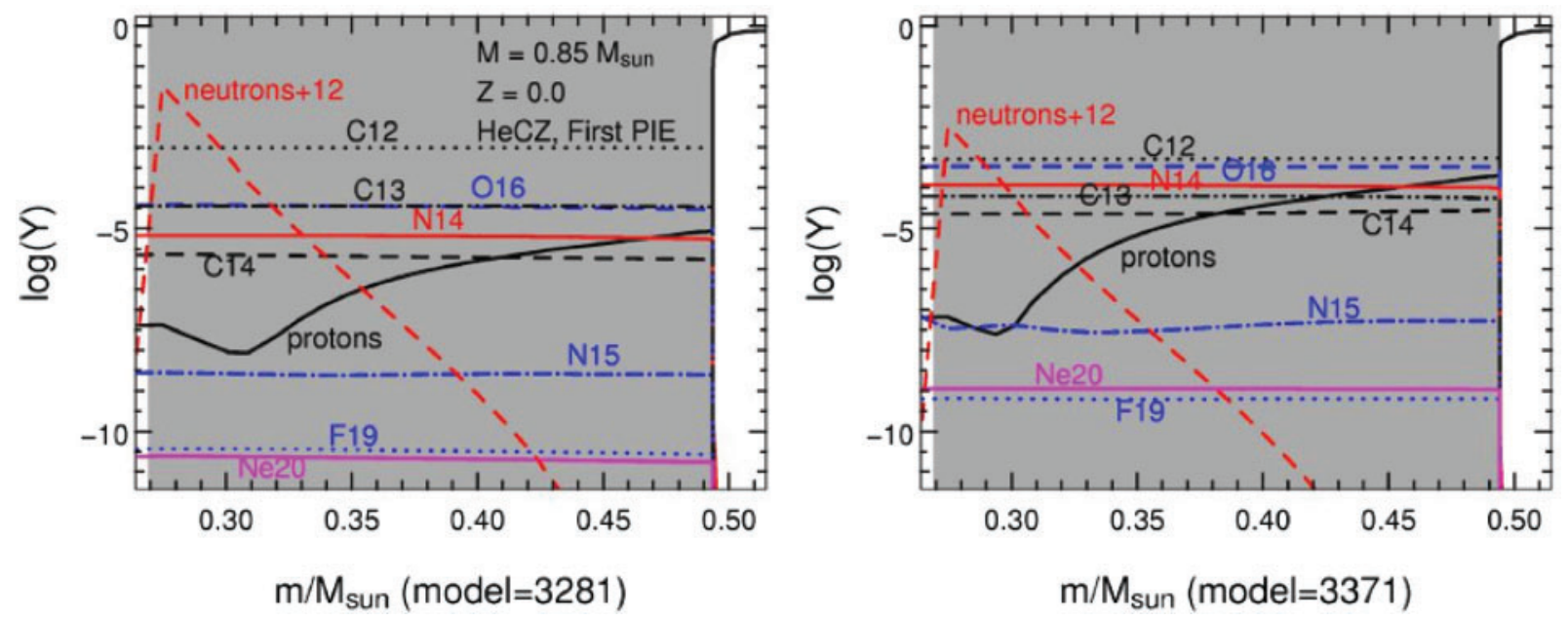

Figure 2 Composition of the convective He/H-burning flash region (grey shading) during the first proton ingestion episode of the DCF for a stellar model of $0.85 \mathrm{M}_{\odot}$ and $Z=0$. The left panel displays the composition near the neutron peak, the right panel is taken $\sim 0.02 \mathrm{yr}$ later. The peak neutron abundance in the left panel corresponds to a neutron density of $2 \times 10^{14} \mathrm{~cm}^{-3}$. (Figure reproduced from figure 6.8 of Campbell 2007.)

${ }^{14} \mathrm{~N}$, via $(n, p)$ reactions. However, in this initial phases of the DCF the abundance of ${ }^{14} \mathrm{~N}$ is still relatively small (abundance by number of the order of $10^{-5}$ ) while it grows over time due to CNO cycling. Thus, the freed neutrons are available for neutron captures that may result in the production of $\mathrm{Fe}$ and heavier elements.

The material exposed to the neutron superburst will be dredged-up to the stellar surface as, after the He/H-burning convective region splits due to the energy generated by $\mathrm{H}-$ burning in the middle of the convective zone, the upper convective region eventually joins the convective envelope (see detailed discussion in, e.g. Fujimoto et al. 2000; Campbell \& Lattanzio 2008).

In the model shown in Figure 2, the neutron density stays above $6 \times 10^{13} \mathrm{~cm}^{-3}$ for $\sim 0.5$ to $1 \mathrm{yr}$, at a temperature $\simeq 2 \times 10^{8} \mathrm{~K}$. The resulting total integrated neutron flux (i.e. the neutron exposure $\tau$ ) is thus $\simeq 250 \mathrm{mbarn}^{-1}$, at least two orders of magnitude larger than typical $s$-process neutron exposures during the AGB phase (see, e.g. Gallino et al. 1998). Since the nuclear network used in the calculations by Campbell (2007) included species up to sulphur, together with a few Fe-peak species, we cannot tell yet which kind of nucleosynthesis may result from this neutron superburst and propose that some kind of $r$-process distribution, together with iron production, may arise from the operation of the neutron superburst. Since this material will become part of the He-rich intershell during the following AGB phase, it will be exposed to the $s$ process. Then, it may be carried to the stellar surface via the third dredge-up and out of the star by the stellar winds, with the signature of both the $r$ and the $s$ processes.

Qualitatively, the large $\tau$ operating on a relatively long timescale during the neutron superburst would not be expected to produce $r$-process elements, but rather a huge amount of $\mathrm{Pb}$, which may make an interesting signature of low-mass Population III stars. However, we have to be careful in considering the large stellar uncertainties connected to the proton ingestion episode. The efficiency of the proton ingestion depends on the physical and numerical treatment of the borders of the convective He/H-burning region. This treatment should account for phenomena such as turbulence, diffusion, rotation, and magnetic fields, which are difficult to describe within one dimensional models. Campbell (2007) used the Schwarzschild criterion to define the border of convective regions. It cannot be excluded that more mixing occurs if other treatments are used, for example, if some form of overshoot is included. This would lead to a shorter and stronger neutron superburst, since ${ }^{13} \mathrm{C}$ would be produced quicker and in larger abundance, however, the poison ${ }^{14} \mathrm{~N}$ would also be produced quicker, thus damping faster the availability of free neutrons.

Finally, we note that because of the use of the Schwarzschild criterion, the models of Campbell \& Lattanzio (2008) do not show any third dredge-up for masses 0.85 and $1 \mathrm{M}_{\odot}$. Again, if the convective borders were treated differently, then the third dredge-up may happen even at such low masses. For example, Stancliffe, Izzard \& Tout (2005) find the third dredge-up to occur for stars of $1 \mathrm{M}_{\odot}$ and metallicities $Z=0.008$ and $Z=0.004$.

\section{Could the Origin of CEMPs $+r$ Stars be Related to the DCF Neutron Superburst?}

Summarizing, in a speculated DCF neutron superburst scenario, CEMP $+r$ stars were originally Population III stars, thus with much lower Fe abundances than observed today. Stars of Population III and mass $\simeq 0.8 \mathrm{M}_{\odot}$, the typical mass of turn-off and giant halo stars, in a binary system with a companion of similar or slighlty higher mass would have become what is observed now as a CEMP $+r$ via mass transfer of material from their companion during its AGB phase. This material could be enriched in Fe and $\mathrm{Eu}$, due to the effect of the DCF neutron superburst, in Ba, 
due to the effect of the $s$ process and third dredge-up during the AGB phase, and in C, N, O, F, and other elements due to the effect of both the DCF and the third dredgeup. In this scenario the $r$ - and the $s$-process components in CEMP $s+r$ stars are naturally correlated, as observed, since both components would occur in the primary star and be dependent on the mass, metallicity, and distance from the companion. Also, the $s$ process would follow the production of the bulk of Eu in the DCF and re-adjust its isotopic ratios as required by the observation.

Finally, in order to explain the high frequency of CEMP $s+r$ stars among Population II stars, we would have to assume the existence of a significant number of Population III stars with initial mass $0.8-1 \mathrm{M}_{\odot}$.

It is obvious that the proposed scenario is highly speculative and further evolutionary and nucleosynthetic calculations with large nuclear networks are needed to find out the possible nucleosynthetic outcomes of the DCF neutron superburst. Only if these calculations provide the possibility of producing $\mathrm{Fe}$ and $\mathrm{Eu}$ in the abundances observed in CEMP $+r$ stars, then we will have to consider carefully a the DCF neutron superburst scenario for CEMP $+r$ stars. In the search for a solution for the CEMP $s+r$ mystery we may have to review our current ideas about the origin of these stars.

\section{Acknowledgments}

M.L. is most grateful to Roberto Gallino for his teaching over many years and for being a constant inspiration to her work. Many thanks for discussion to Sara Bisterzo, as well as Onno Pols, Rob Izzard and the rest of the stellar evolution group in Utrecht. M.L. is grateful to NWO for a VENI fellowship and to Monash University for a Monash Research Fellowship.

\section{References}

Aoki, W., Honda, S., Beers, T. C. \& Sneden, C., 2003a, ApJ, 586, 506

Aoki, W. et al., 2003b, ApJ, 592, L67
Bisterzo, S., 2007, PhD Thesis, Universitá di Torino

Campbell, S. W., 2007, PhD Thesis, Monash University

Campbell, S. W. \& Lattanzio, J. C., 2008, A\&A, 490, 769

Cohen, J. G., Christlieb, N., Qian, Y.-Z. \& Wasserburg, G. J., 2003, ApJ, 588, 1082

Fujimoto, M. Y., Iben, I. J. \& Hollowell, D., 1990, ApJ, 349, 580

Fujimoto, M. Y., Ikeda, Y. \& Iben, I. J., 2000, ApJ, 529, L25

Gallino, R., Arlandini, C., Busso, M., Lugaro, M., Travaglio, C., Straniero, O., Chieffi, A. \& Limongi, M., 1998, ApJ, 497, 388

Hollowell, D., Iben, I. J. \& Fujimoto, M. Y., 1990, ApJ, 351, 245

Ivans, I. I., Sneden, C., Gallino, R., Cowan, J. J. \& Preston, G. W., 2005, ApJ, 627, L145

Jonsell, K., Barklem, P. S., Gustafsson, B., Christlieb, N., Hill, V., Beers, T. C. \& Holmberg, J., 2006, A\&A, 451, 651

Karakas, A. \& Lattanzio, J. C., 2007, PASA, 24, 103

Lucatello, S., Tsangarides, S., Beers, T. C., Carretta, E., Gratton, R. G. \& Ryan, S. G., 2005, ApJ, 625, 825

Lugaro, M. et al., 2008, A\&A, 484, L27

Mac Low, M. M., 2008, ASPC, 387, 148

Megeath, S. T., Gaidos, E., Hester, J. J., Adams, F. C., Bally, J., Lee, J.-E. \& Wolk, S., 2008, ASPC, 384, 393

Picardi, I., Chieffi, A., Limongi, M., Pisanti, O., Miele, G., Mangano, G. \& Imbriani, G., 2004, ApJ, 609, 1035

Qian, Y.-Z. \& Wasserburg, G. J., 2003, ApJ, 588, 1099

Schuler, S. C., Cunha, K., Smith, V. V., Sivarani, T., Beers, T. C. \& Lee, Y. S., 2007, ApJ, 667, L81

Sneden, C., Cowan, J. J., Lawler, J. E., Burles, S., Beers, T. C. \& Fuller, G. M., 2002, ApJ, 566, L25

Sneden, C., Preston, G. W., McWilliam, A. \& Searle, L., 1994, ApJ, 431, L27

Stancliffe, R. J., Izzard, R. G. \& Tout, C. A., 2005, MNRAS, 356, L1

Suda, T. et al., 2008, PASJ, 60, 1159

Thompson, I. B. et al., 2008, ApJ, 677, 556

Travaglio, C., Galli, D., Gallino, R., Busso, M., Ferrini, F. \& Straniero, O., 1999, ApJ, 521, 691

Vanhala, H. A. T. \& Cameron, A. G. W., 1998, ApJ, 508, 291

Wanajo, S., Nomoto, K., Iwamoto, N., Ishimaru, Y. \& Beers, T. C., 2006, ApJ, 636, 842

Zijlstra, A. A., 2004, MNRAS, 348, L23 